\title{
LOCAL GRAY WORLD METHOD FOR SINGLE IMAGE DEHAZING
}

\author{
Vedran Stipetić and Sven Lončarić \\ Faculty of Electrical Engineering and Computing, \\ University of Zagreb, Zagreb, Croatia
}

\begin{abstract}
Images taken outdoors are often degraded by atmospheric conditions such as fog and haze. These degradations can reduce contrast, blur edges, and reduce saturation of images. In this paper we propose a new method for single image dehazing. The method is based on an idea from color constancy called the gray world assumption. This assumption states that the average values of each channel in a picture are the same. Using this assumption and a haze degradation model we can quickly and accurately estimate the haze thickness and recover a haze free image. The proposed method is validated on a synthetic and natural image dataset and compared to other methods. The experimental results have shown that the proposed method provides comparable results to other dehazing methods.
\end{abstract}

\section{KEYWORDS}

image restoration, image dehazing

\section{INTRODUCTION}

Images taken outdoors are often degraded by atmospheric conditions such as fog and haze. These degradations can reduce contrast, blur edges, and reduce saturation of images. As a result, many computer vision algorithms work worse in hazy conditions than in optimal weather conditions. For this reason the problem of image dehazing is an important one. Since in many real life applications it is only possible to have a single hazy image of an area, without haze free reference image, depth information, multiple images taken with different polarizations or similar additional information, there has been much effort in developing algorithms capable of removing haze from a single image.

\section{Prior Work}

Most single image dehazing models are based on the physical description of how haze degrades an image. This description is derived for instance in [1]. It is given as

$$
I^{c}=I_{0}^{c} t+(1-t) A
$$

Where $\mathrm{c}$ denotes the channel, so $\mathrm{c}$ is either R, G or B. I denotes the intensity in that channel in the observed image, $\mathrm{I}_{0}$ denotes the intensity of the non-degraded image, A denotes the airlight, or the illumination dispersed by the haze and $\mathrm{t}$ is the transmission map that balances between scene radiance and airlight. Common assumptions made about the airlight $\mathrm{A}$ are that it is constant on the entire image and sometimes that it is the same in all three channels. The transmission map depends on the depth of the scene and haze density and is given by the formula 


$$
t=\int e^{\beta d(x)} d x
$$

Here the scene depth is denoted by $d(x)$ and haze density by $\beta$. Mathematically this results in a system of 3 equations with 5 unknowns, the intensities of each channel, airlight and transmission, for each pixel. In order to solve this underdetermined system, it is necessary to introduce some prior knowledge. The most common prior used in dehazing is the dark channel prior first introduced by $\mathrm{He}$ et al in [2]. This prior assumes that in any local patch of a natural image, there exists a pixel with very low intensity in at least one of its channels. Then, in a hazy image the brightness of this dark channel in a patch must come from the haze, and so can be used to estimate transmission. The dark channel prior (sometimes abbreviated to DCP) is also widely used to estimate haze, by taking the most haze opaque pixels in the image. Many other papers built on the dark channel prior approach such as [3] where guided filter is used to smooth out the initial rough transmission estimate.

A different approach called color attenuation prior (sometimes abbreviated to CAP) is introduced in [4]. There, the authors use the fact that as depth increases, the saturation decreases and the intensity increases. So the difference between saturation and value components can be used to estimate the transmission. This is done using a linear model, with coefficients determined using machine learning. Once the transmission map is estimated this way, the pixels estimated to be the deepest in the image can be used to estimate the airlight A.

A third approach is the variational approach where parameters are determined by minimizing a functional derived from different priors. For example in [5] the generalized total variation prior is used, based in the assumption that the transmission map is piecewise smooth. In [6] the prior used was built upon ideas from computational color constancy, namely gray world assumption, which will also be used in this paper.

Although all of these methods have their advantages, they also have their shortcomings. The variational methods are usually slow, so they cannot be used in real time systems. Dark channel prior-based methods often exhibit halo effects near very bright objects in the image and color attenuation prior has trouble with scenes that are not naturally saturated, such as gray roads or other urban areas. Because of this we propose a new method, with the goal of overcoming these shortcomings.

\section{Proposed METhod}

In this paper we propose a new method for single image dehazing. First, the airlight is estimated using the technique described in [2]. Then a coarse transmission map is estimated using a local version of the gray world prior. The transmission map is then refined using guided image filtering [3].

\subsection{Gray World Assumption}

The gray world assumption is a well-known prior in the area of computational color constancy. It is usually stated assuming that the average value in each of the channels is the same.

$$
\frac{1}{N} \sum R_{i}=\frac{1}{N} \sum G_{i}=\frac{1}{N} \sum B_{i}
$$

Another assumption that is sometimes made when using the gray world prior is that the average value is close to one half. This assumption is also used in [6]. 


\subsection{Estimating Airlight}

The method for estimating airlight is taken from the dark channel prior [2], but will also be described here for the sake of completeness. Since it is assumed that airlight has high brightness, it is larger than the dark channel in most pixels. Also, as depth increases, the transmission goes to zero, and so the pixels with large depth are very similar in color to airlight. Because of this, if we take a small number of brightest pixels in the dark channel, they will usually approximate airlight well, as they are the most haze opaque ones. In particular we take the $0.1 \%$ brightest pixels in the dark channel and then take the pixel with highest intensity. The intensity of that pixel is set to be the airlight.

\subsection{Estimating Transmission}

In order to get a coarse estimate of transmission, we will use a local gray world assumption. First we assume that transmission is constant in a small $\mathrm{N}^{*} \mathrm{~N}$ patch around a pixel. Then, on that patch we have

$$
\sum(I-A)=\left(\sum\left(I_{0}-A\right)\right) t
$$

So, after using the local gray world assumption that the average value of $I_{0}$ on that patch is 0.5 , we get the expression for transmission

$$
t=\frac{\frac{1}{N^{2}} \sum(I-A)}{\frac{1}{2}-A}
$$

This can be done for all three channels. Empirically, we have determined that the best way to combine the information from all the different channels is to choose as the value of transmission in that pixel the value calculated from the channel with smallest average in the patch. After the transmission has been estimated in all pixels it is normalized by dividing the entire transmission map by the largest value that appears in the estimation. This is done to avoid physically unreasonable transmission estimates, such as the ones larger than 1.

This estimation is then refined using guided image filtering reduce the blockiness caused by the patch based approach to estimating transmission and avoid halo effects around edges in the restored image.

Once both airlight and transmission are estimated, the dehazed image is reconstructed from the equation:

$$
I_{0}=\frac{I-A}{t}+A
$$

For purposes of numerical stability, when $\mathrm{t}$ is smaller than 0.05 it is set to 0.05 .

\section{EXPERIMENTAL RESULTS}

In this section we present our experimental results and compare the proposed local gray world method to dark channel prior and color attenuation prior methods. The tests are done on both natural hazy images with unknown ground truth, and on synthetic indoor images with ground truth known. The synthetic images were taken from the RESIDE dataset [7]. All images were first 
rescaled to $400 * 400$ pixel format to ensure that patch sizes remain consistent across different images.

\subsection{Qualitative Results}

Qualitative results are ones judged visually on natural images with no known ground truth. These results are important because they judge the performance of dehazing on real haze degradation, which does not fully follow the simplified model from [1].
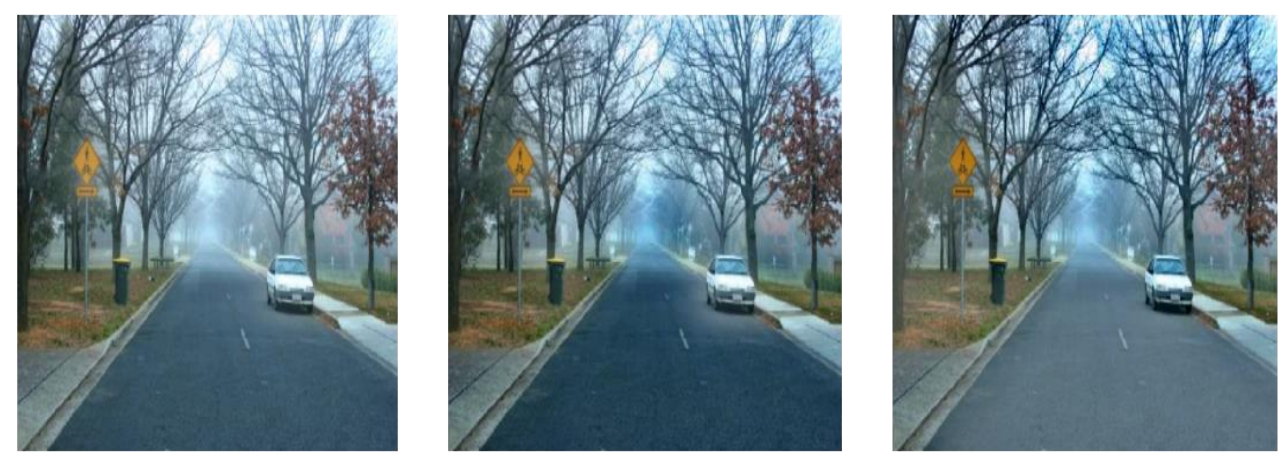

Figure 1. left - result of CAP, middle result of DCP, right result of our method.

In Figure 1 we can see the results of all three algorithms. The proposed local gray world method shows better properties of dehazing in the deeper parts of the image than color attenuation prior and has none of the halo effects caused by white objects in the dark channel prior. The color of the road is also better restored, as it is too dark in both of the other images. Figure 2 shows the original hazy version of the road image, and in Figure 3 we can see the estimated transmission maps of all three algorithms.

In Figure 4 we can see the results when applied to another outdoor image. Once again, the dark channel prior and our local gray world method give good results. The dark channel method again has some haloing issues, and the gray world method has too large contrast near the tops of mountains. This happens because of the sudden jump in color between mountains and the sky where the gray world assumption is not close to being valid.

In Figure 5 we see results applied on a hazy image of a field full of hay bales. In this image the oversaturation effect of our method is especially pronounced. The dark channel prior approach also seems to have removed the haze near the camera much better.

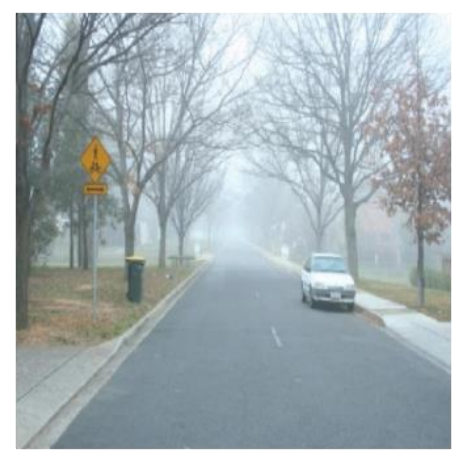

Figure 2. Original road image. 

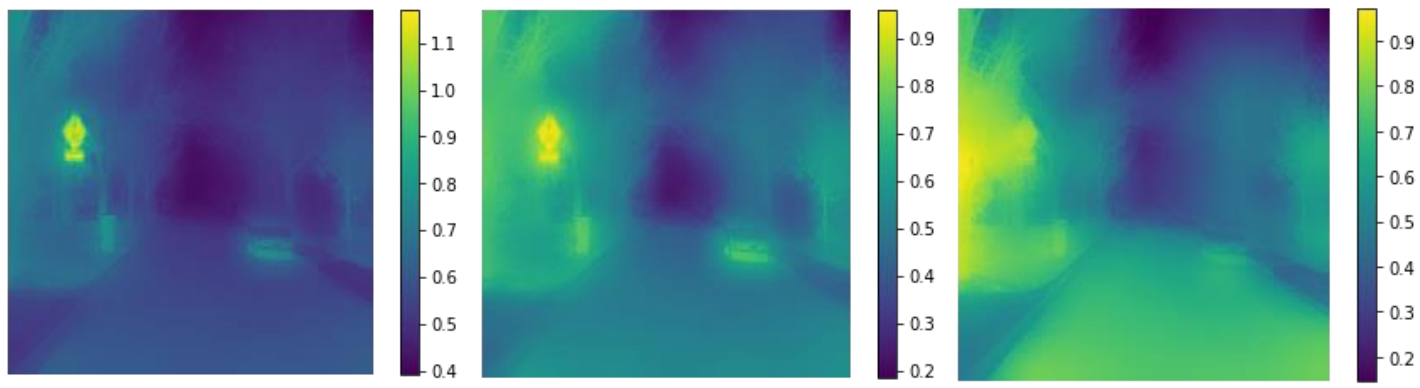

Figure 3. Estimated transmissions: left CAP, middle DCP, right proposed method.
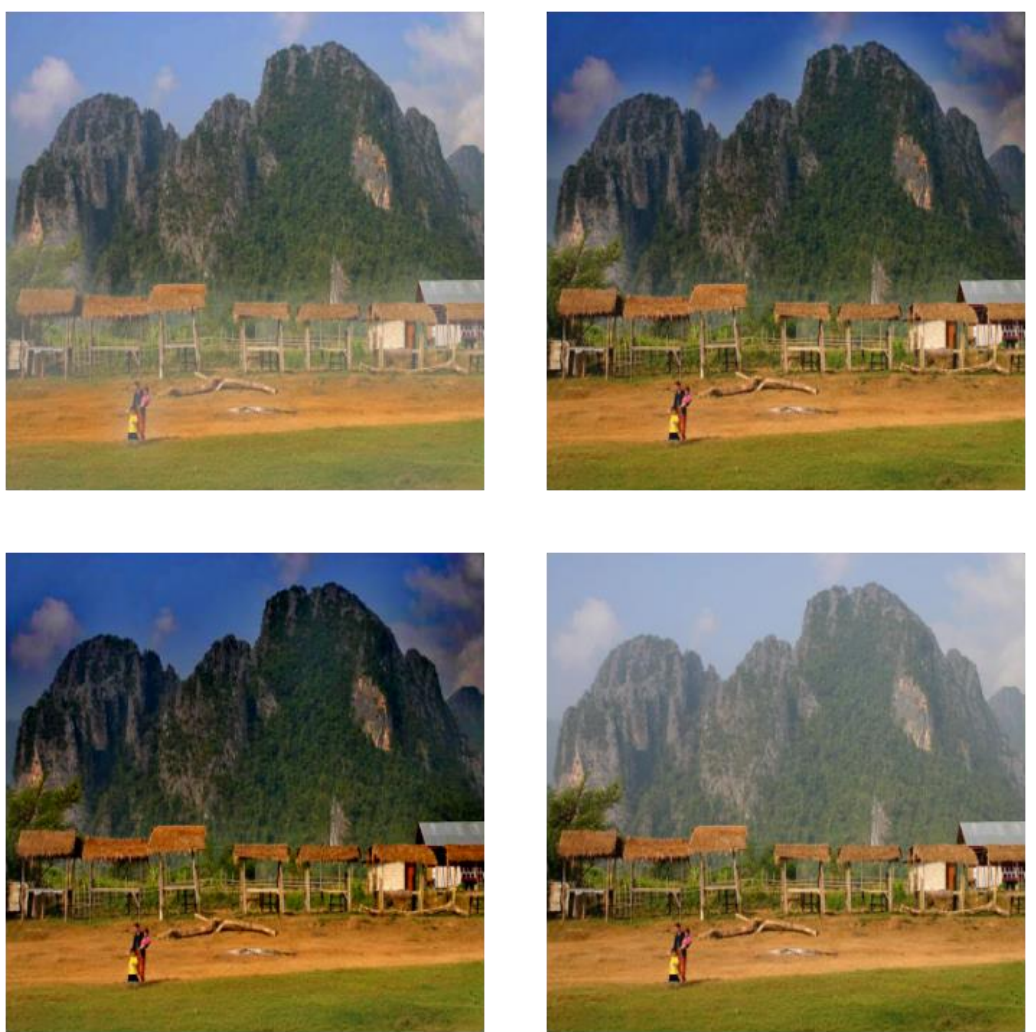

Figure 4. Top left, CAP result, top right DCP result, bottom left local gray world result bottom right original image. 

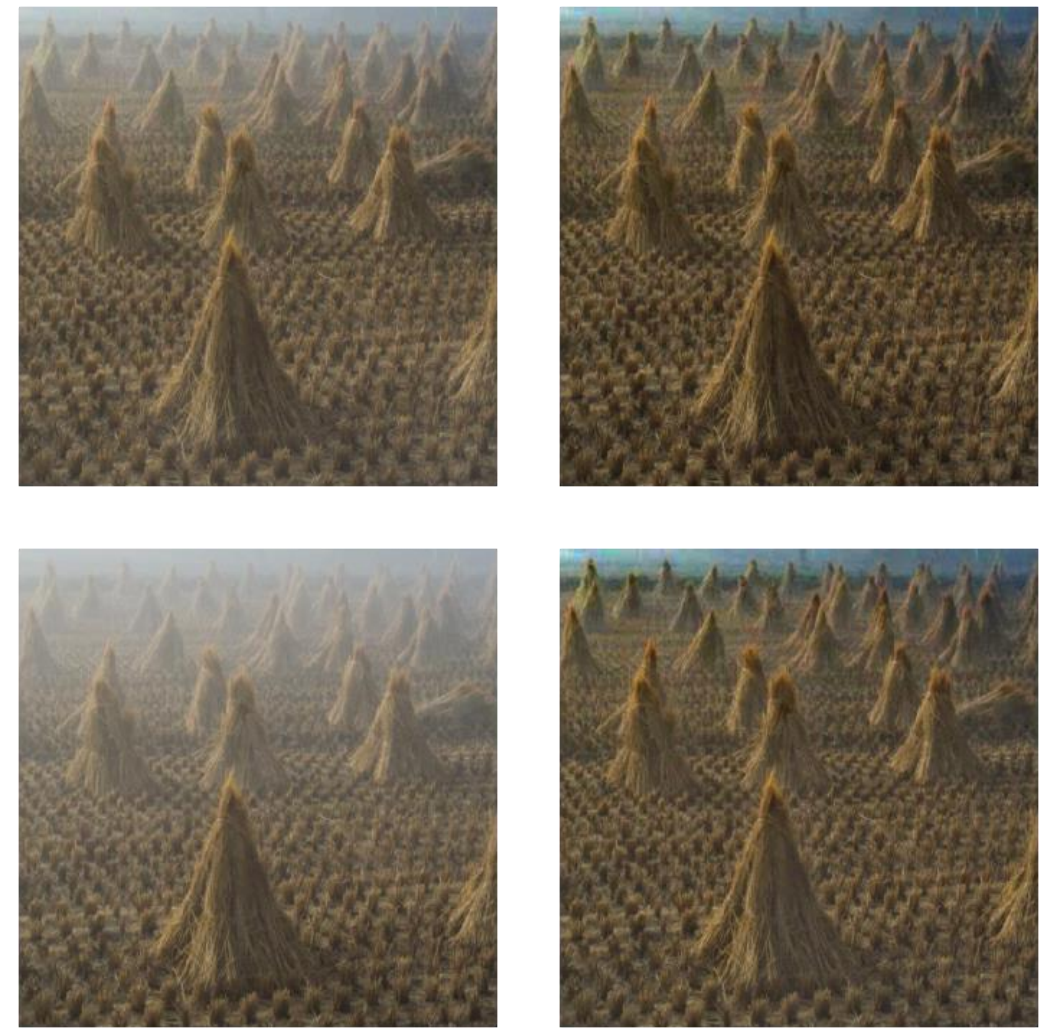

Figure 5. Top left CAP result, top right DCP result, bottom left original hazy image, bottom right local gray

\subsection{Quantitative Results} world result.

In this section we present quantitative results of dehazing using the proposed local gray world algorithm and take dark channel prior and color attenuation prior as benchmarks to compare against. The images were taken from RESIDE dataset [7] that is commonly used to evaluate dehazing algorithms. For each image and each algorithm we calculated structural similarity index, peak signal to noise ratio and mean square error compared to ground truth. The results are presented in tables 1, 2 and 3. SSIM stands for structural similarity index, PSNR stands for peak signal to noise ratio and MSE stands for mean squared error. We can see that according to all of the metrics on both images, color attenuation prior seems to be the best, while the proposed method seems to be worse than both others on the synthetic data. The actual dehazed images and originals can be seen on Figures 6 to 9. In Table 3 we present the averaged results on 100 randomly chosen images from the RESIDE dataset. We can see that on avarage the dark channel prior seems to be the best according to all metrics. We can see in the Figures that while the local gray world method seem to be good at removing the haze, it also oversaturates colors and increases contrast too much resulting in poor quantitative results.

Table 1. Quantitative evaluation on Hallway image. For MSE lower numbers are better, for SSIM and PSNR higher numbers are better.

\begin{tabular}{|l|l|l|l|}
\hline \multicolumn{1}{|c|}{ Hallway } & \multicolumn{1}{|c|}{ CAP } & \multicolumn{1}{c|}{ DCP } & \multicolumn{1}{c|}{ Local gray world } \\
\hline SSIM & 0.92591 & 0.89616 & 0.88816 \\
\hline PSNR & 20.05890 & 17.37561 & 16.13917 \\
\hline MSE & 0.00986 & 0.01829 & 0.02432 \\
\hline
\end{tabular}


Table 2. Quantitative evaluation on NYU image. For MSE lower numbers are better, for SSIM and PSNR higher numbers are better.

\begin{tabular}{|l|l|l|l|}
\hline \multicolumn{1}{|c|}{ NYU } & \multicolumn{1}{c|}{ CAP } & \multicolumn{1}{c|}{ DCP } & \multicolumn{1}{c|}{ Local gray world } \\
\hline SSIM & 0.92528 & 0.92519 & 0.89771 \\
\hline PSNR & 17.10202 & 17.68711 & 16.06112 \\
\hline MSE & 0.01948 & 0.01703 & 0.02476 \\
\hline
\end{tabular}
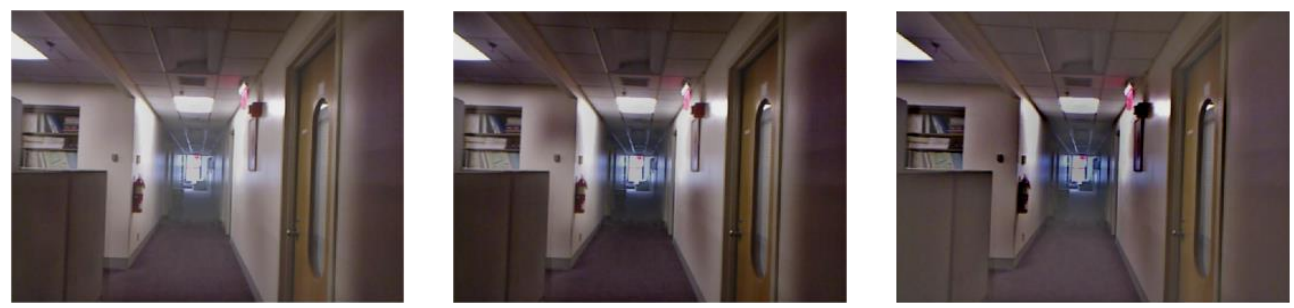

Figure 6. Hallway dehazing results. From left to right CAP, DCP and local gray world.
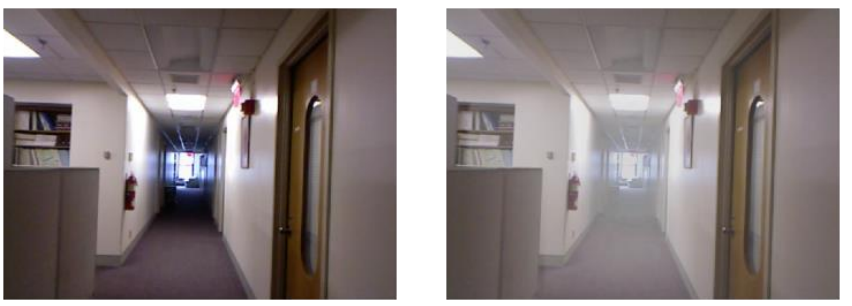

Figure 7. Ground truth (left) and artificially hazy hallway image.
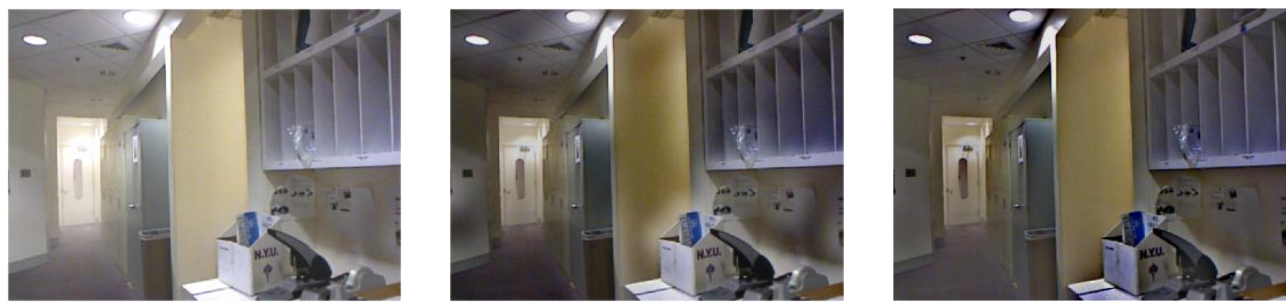

Figure 8. NYU dehazing results. From left to right: CAP, DCP local gray world.
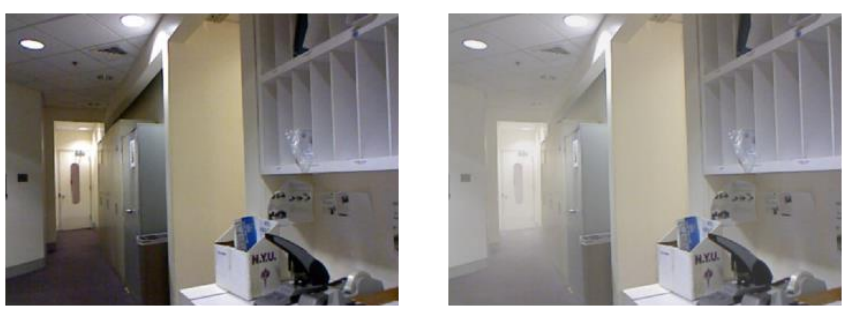

Figure 9. Ground truth (left) and artificially hazy NYU image.

Table 3. Average results for 100 randomly chosen images from the RESIDE indoor dataset. For MSE lower numbers are better, for SSIM and PSNR higher numbers are better.

\begin{tabular}{|l|l|l|l|}
\hline & \multicolumn{1}{|c|}{ CAP } & \multicolumn{1}{|c|}{ DCP } & \multicolumn{1}{c|}{ LOCAL GRAY WORLD } \\
\hline SSIM & 0.77925 & 0.85182 & 0.76476 \\
\hline PSNR & 14.35013 & 17.61810 & 13.90976 \\
\hline MSE & 0.06335 & 0.04891 & 0.09311 \\
\hline
\end{tabular}




\section{CONCLUSION AND FUTURE WORK}

The proposed local gray world dehazing method presented in this paper performs comparably to benchmark methods in image dehazing on synthetic data and sometimes even better on natural images. As it only uses low level image statistics it is also a fast and computationally efficient method. Since the proposed method uses patches around each pixel, it has the same complexity as other methods discussed in the paper, however, it is slightly faster since it uses fewer operations per patch. The proposed method can be especially useful when an image contains large gray surfaces, such as roads or buildings, as we showed in the example in Figure 1. This leads us to conclude that this is an interesting direction for research. Another potential novelty that the local gray world method brings is the information about transmission in all three channels. In this paper we conformed to the norm and used the same transmission in all three channels, but this could be an interesting direction for future experiments.

\section{REFERENCES}

[1] S. K. Nayar and S. G. Narasimhan, "Vision in bad weather", in Proc. IEEE International Conference on Computer Vision (ICCV), vol. 2. Sep. 1999, pp 820-827

[2] K. He, J. Sun, X. Tang, "Single image haze removal using dark channel prior" IEEE Transactions on Pattern Analysis and Machine Intelligence, vol. 33, no. 12, pp. 2341-2353, Dec. 2011.

[3] K. He, J. Sun, X. Tang, "Guided image filtering", IEEE Transactions on Pattern Analysis and Machine Intelligence, vol. 35, no. 6, pp 1397 - 1409, Jun. 2013

[4] Q. Zhu, J. Mai, L. Shao "A Fast Single Image Haze Removal Algorithm Using Color Attenuation Prior”, IEEE Transactions on Image Processing, vol. 24, no. 11, pp. 3522-3533, Nov 2015

[5] Y. Gu, X. Yang, Y Gao, “A Novel Total Generalized Variation Model for Image Dehazing”, Journal of Mathematical Imaging and Vision, vol. 61 1329-1341, 2019

[6] A. Galdran, J. Vasquez-Corral, D. Pardo and M. Bertalmio, "Enhanced Variational Image Dehazing", SIAM Journal of Imaging Sciences, vol. 8, issue 3, pp 1519-1546, July 2015

[7] B. Li, W. Ren, D. Fu, D. Tao, D. Feng, W. Zeng, Z. Wang, "Benchmarking Single-Image Dehazing and Beyond", IEEE Transactions on Image Processing, vol. 28, no. 1, pp 492-505, Jan. 2019 